\title{
Minimum BER Power Allocation for OFDM-based Cognitive Radio Networks
}

\author{
Ding Xu and Qun Li \\ Wireless Communication Key Lab of Jiangsu Province, Nanjing University of Posts and Telecommunications, \\ Nanjing, China. \\ [e-mail: xuding.bupt@gmail.com, liqun@njupt.edu.cn] \\ *Corresponding author: Ding Xu
}

Received March 15, 2015; revised May 11, 2015; accepted June 8, 2015;

published July 31, 2015

\begin{abstract}
In this paper, the optimal power allocation algorithm that minimizes the aggregate bit error rate (BER) of the secondary user (SU) in a downlink orthogonal frequency division multiplexing (OFDM) based cognitive radio (CR) system, while subjecting to the interference power constraint and the transmit power constraint, is investigated under the assumption that the instantaneous channel state information (CSI) of the interference links between the secondary transmitter and the primary receiver, and between the primary transmitter and the secondary receiver is perfectly known. Besides, a suboptimal algorithm with less complexity is also proposed. In order to deal with more practical situations, we further assume that only the channel distribution information (CDI) of the interference links is available and propose heuristic power allocation algorithms based on bisection search method to minimize the aggregate BER under the interference outage constraint and the transmit power constraint. Simulation results are presented to verify the effectiveness of the proposed algorithms.
\end{abstract}

Keywords: Cognitive radio, OFDM, power allocation, bit error rate, channel distribution information.

This work was supported by the National High-Tech R\&D Program (863 Program 2015AA01A705 and 973 Program 2013CB329005), the National Natural Science Foundation of China (Grant No. 61401218) and NUPTSF (Grant No. NY214062 and NY213086). 


\section{Introduction}

Cognitive radio (CR), as a promising technique to solve the problem of spectrum scarcity, has attracted lots of research attentions over the past dozen years [1]. In CR networks, licensed users, also known as primary users (PUs), and unlicensed users, also known as secondary users (SUs), coexist on the spectrum bands licensed to the PUs under the condition that the quality of service (QoS) of the PUs is not affected unduly. There are three models for the SUs to share the spectrum with the PUs: interweaved, overlay and underlay [2]. Similar to [3]-[5], we focus on the underlay model, in which the SUs can transmit simultaneously with the PUs over the same spectrum as long as the interference from the SUs to the PUs is below an acceptable limit.

On the other hand, orthogonal frequency division multiplexing (OFDM) is regarded as a promising candidate transmission technology for CR networks, because of its high transmission efficiency and flexibility in allocating radio resources. Therefore, OFDM-based CR networks have drawn a lot of attentions. In particular, the resource allocation problems in OFDM-based CR networks are hot research topics. In [4], power allocation algorithms were proposed for an OFDM-based CR network to maximize the SU sum rates under the interference power constraint only. In [5], efficient power allocation algorithms to maximize the SU sum rates under the interference power constraint as well as the transmit power constraint were proposed. [6] studied the power allocation problem to maximize the SU sum rates under the PU protection criterion expressed through a utility function. In [7], a constant interference strategy was proposed to allocate power to subcarriers such that the subcarriers are subject to the same interference. In [8], a fast and efficient power allocation algorithm for the OFDM-based CR network was proposed based on the parallel-shift property of water-filling. [9] proposed to utilize the gradient descent method to allocate power to subcarriers in OFDM-based CR networks to maximize the SU sum rates. In [10], the optimal power allocation strategy to maximize the SU sum rates under the PU rate loss constraint was proposed. In [11], two power allocation schemes with opportunistic subchannel access were proposed, respectively, to maximize the sum rates of the SU and the sum rates of the SU and the PU. In [12], a worst-case robust distributed power allocation scheme based on a non-cooperation game framework was proposed for an OFDM-based CR networks. In [13], the power allocation problems for a two-way OFDM-based CR network to maximize the ergodic sum rates of the SUs were investigated. In [14], a low-complex suboptimal power allocation algorithm was proposed for an OFDM-based CR network to maximize the SU sum rates.

The objectives of the above studies are to maximize the SU rate. However, the SU rate is clearly not the only performance indicator for the CR networks. Other important performance indicators include energy efficiency [15-17], outage probability [18], [19] and bit error rate (BER) (or symbol error probability, SEP) [20], [21]. In this context, BER, which is an important performance indicator in determining the transmission quality of the SU, has received much attention. For example, [20] proposed the optimal power allocation algorithms to minimize the average BER of the SU subject to various transmit power and interference power constraints. In [21], the expressions for the minimum average BER of the SU under either peak or average interference power constraints were derived based on the proposed optimal power allocation strategies. In [22], the expression for the average BER of an uplink $\mathrm{CR}$ network with opportunistic scheduling was derived based on the assumption that the SU 
which causes the minimum interference to the PU is selected for transmission. In [23], the authors proposed to choose the SU with the best channel quality for transmission and derived the expression for the average BER which is shown to outperform the result in [22]. In [24], the BER of the SU with difference antenna selection scheme was analyzed. In [25], closed-form expression for the BER of the multi-hop SUs under Rayleigh fading was derived. However, existing studies on BER of the SU, such as [20-25], focus on single channel non-OFDM-based CR networks. To the best of our knowledge, the problem of power allocation in OFDM-based CR networks targeting at minimizing the BER of the SU has not been touched yet in the existing literature.

Therefore, in this paper, an OFDM-based CR network is considered to coexist with a PU network. Instead of using the SU sum rates as the performance metric, minimizing the aggregate BER of the SU is considered to be the objective of the power allocation problem. The main contributions are:

1) Assuming that the instantaneous channel state information (CSI) of the interference links between the secondary transmitter and the primary receiver, and between the primary transmitter and the secondary receiver is perfectly known at the SU, we derive the optimal power allocation algorithm under the interference power constraint and the transmit power constraint. Besides, a suboptimal algorithm with less complexity is also provided. The performance of the proposed algorithms is compared with three existing reference algorithms, i.e., the uniform power allocation algorithm, the conventional water-filling algorithm [26] and the optimal sum-rate algorithm [5]. It is shown that the proposed algorithms achieve much lower aggregate BER than that of the reference algorithms.

2) Assuming that only the channel distribution information (CDI) of the interference links is available at the SU, we propose power allocation algorithms based on bisection search method to minimize the aggregate BER under the interference outage constraint and the transmit power constraint. It is shown that the proposed algorithms can well protect the PU QoS in terms of interference outage probability with only CDI available. It is also shown that the performance of the SU with only CDI drops compared to the case of perfect CSI.

The rest of the paper is organized as follows. The system model is presented in Section 2. With the perfect instantaneous CSI of the interference links known at the SU, the corresponding power allocation algorithms to minimize the aggregate BER of the SU under the interference power constraint and the transmit power constraint are given in Section 3. With the CDI of the interference links known at the SU, the corresponding power allocation algorithms to minimize the aggregate BER of the SU under the interference outage constraint and the transmit power constraint are given in Section 4. Simulation results are provided in Section 5 to verify the proposed algorithms. Section 6 concludes the paper.

\section{System Models}

In this paper, an OFDM-based CR network, where a pair of secondary transmitter and secondary receiver share the same spectrum with the PU on $N$ subcarriers, is considered ${ }^{1}$. The

\footnotetext{
${ }^{1}$ In this paper, we focus on the problem of power allocation and thus subcarrier allocation is assumed to have been executed. It is noted that once the subcarriers are allocated to the SUs, the multi-user system can be viewed virtually as a single user multi-subcarrier system as shown in [27]. Thus, without loss of generality, we consider only one pair of SUs to emphasize our main focus on power allocation.
} 
instantaneous channel power gains on subcarrier $n$ from the secondary transmitter to the secondary receiver, the secondary transmitter to the primary receiver, and the primary transmitter to the secondary receiver are denoted by $g_{s s}^{n}, g_{s p}^{n}$ and $g_{p s}^{n}$, respectively. All the channels involved are assumed to be independent block fading channels. The noises power is denoted by $\sigma^{2}$. It is assumed that the SU has perfect CSI of its own communication link, i.e., the instantaneous channel power gains, $g_{s s}^{n}$, for all $N$ subcarriers. In practice, $g_{s s}^{n}$ can be obtained at the SU by classic channel estimation and feedback mechanisms. As for the CSI of the interference links, i.e., the instantaneous channel power gains, $g_{s p}^{n}$ and $g_{p s}^{n}$, for all $N$ subcarriers, two scenarios are considered. In the first scenario in Section 3, we assume perfect knowledge of $g_{s p}^{n}$ and $g_{p s}^{n}$ at the SU. In practice, $g_{s p}^{n}$ can be obtained by cooperation between the SU and the PU. For example, the PU can estimate $g_{s p}^{n}$ and then feed it back to the SU. Similarly, $g_{p s}^{n}$ can be obtained at the SU by estimating the received signal power from the primary transmitter. In the second scenario in Section 4 , a more practical situation is considered where only the CDI of $g_{s p}^{n}$ and $g_{p s}^{n}$ is available at the SU. It is noted that long-time channel measurements can be conducted to obtain such CDI.

\section{Power Allocation with Perfect CSI}

In this section, with the perfect CSI of the interference links available at the SU, the problem of power allocation for the SU to minimize the aggregate BER, under the interference power constraint and the transmit power constraint, is studied. The power allocation problem is formulated as follows

$$
\begin{aligned}
\mathrm{P} 1: \min _{P_{n} \geq 0} 1-\prod_{n=1}^{N}\left(1-\operatorname{ber}\left(\gamma_{n}\right)\right) \\
\text { s.t. } \sum_{n=1}^{N} g_{s p}^{n} P_{n} \leq \bar{I}, \\
\quad \sum_{n=1}^{N} P_{n} \leq \bar{P},
\end{aligned}
$$

where $P_{n}$ is the transmit power of the $\mathrm{SU}$ on subcarrier $n, \bar{I}$ is the prescribed interference power limit, $\bar{P}$ is the prescribed transmit power limit, $\gamma_{n}=g_{s s}^{n} P_{n} /\left(\sigma^{2}+g_{p s}^{n} T_{n}\right)$ is the signal to interference and noise ratio (SINR) at the SU on subcarrier $n, T_{n}$ is the transmit power of the PU on subcarrier $n$, and $\operatorname{ber}\left(\gamma_{n}\right)$ is BER on subcarrier $n$ as a function of $\gamma_{n}$. Assume Gray-coded square $M$-ary quadrature amplitude modulation (QAM) scheme is adopted, then we have [28]

$$
\operatorname{ber}\left(\gamma_{n}\right)=\sum_{j=1}^{\varphi(M)} G_{M, j} Q\left(\sqrt{g_{M, j} \gamma_{n}}\right),
$$

where $M$ is the constellation size, $\varphi(M)$ is the number of terms for summation, $Q(x)$ is the Gaussian probability integral defined as $Q(x)=\frac{1}{\sqrt{2 \pi}} \int_{x}^{\infty} e^{-\frac{t^{2}}{2}} d t$, and $\left(G_{M, j}, g_{M, j}\right)$ is a pair of modulation mode dependent constants given in [28]. Considering the fact that minimizing $1-\prod_{n=1}^{N}\left(1-\operatorname{ber}\left(\gamma_{n}\right)\right)$ is equivalent to maximizing $\prod_{n=1}^{N}\left(1-\operatorname{ber}\left(\gamma_{n}\right)\right), \mathrm{P} 1$ is equivalent to the following problem 


$$
\max _{P_{n} \geq 0} \prod_{n=1}^{N}\left(1-\operatorname{ber}\left(\gamma_{n}\right)\right)
$$

$$
\text { s.t. constraints }(2),(3) \text {. }
$$

Since $\ln (x)$ is a monotonically increasing function of $x$, maximizing $\prod_{n=1}^{N}\left(1-\operatorname{ber}\left(\gamma_{n}\right)\right)$ is equivalent to maximizing $\ln \prod_{n=1}^{N}\left(1-\operatorname{ber}\left(\gamma_{n}\right)\right)$. Thus, one can show that the above problem has the same solution as the one for the following problem

$$
\begin{array}{r}
\mathrm{P} 2: \max _{P_{n} \geq 0} \sum_{n=1}^{N} \ln \left(1-\operatorname{ber}\left(\gamma_{n}\right)\right) \\
\text { s.t. constraints }(2),(3) .
\end{array}
$$

It can be easily verified that the objective function in (6) is concave and the constraints (2) and (3) are affine functions with respect to $P_{n}$. Therefore, P2 is concave and can be solved by convex optimization [29].

The Lagrangian of $\mathrm{P} 2$ is expressed as

$$
\begin{aligned}
\mathcal{L}= & \sum_{n=1}^{N} \ln \left(1-\sum_{j=1}^{\varphi(M)} G_{M, j} Q\left(\sqrt{\frac{g_{M, j} g_{s s}^{n} P_{n}}{\sigma^{2}+g_{p s}^{n} T_{n}}}\right)\right) \\
& -\lambda\left(\sum_{n=1}^{N} P_{n} g_{s p}^{n}-\bar{I}\right)-\mu\left(\sum_{n=1}^{N} P_{n}-\bar{P}\right),
\end{aligned}
$$

where $\lambda$ and $\mu$ are the dual variables associated with constraints (2) and (3), respectively. It is observed that $\mathcal{L}$ can be rewritten as

$$
\mathcal{L}=\sum_{n=1}^{N} \mathcal{L}_{n}+\lambda \bar{I}+\mu \bar{P}
$$

where

$$
\mathcal{L}_{n}=\ln \left(1-\sum_{j=1}^{\varphi(M)} G_{M, j} Q\left(\sqrt{\frac{g_{M, j} g_{s s}^{n} P_{n}}{\sigma^{2}+g_{p s}^{n} T_{n}}}\right)\right)-\left(\lambda g_{s p}^{n}+\mu\right) P_{n} .
$$

Then, the Lagrange dual function of the problem is expressed as

$$
\begin{aligned}
\mathcal{G} & =\max _{P_{n} \geq 0} \mathcal{L} \\
& =\sum_{n=1}^{N} \mathcal{G}_{n}+\lambda \bar{I}+\mu \bar{P},
\end{aligned}
$$

where

$$
\mathcal{G}_{n}=\max _{P_{n} \geq 0} \mathcal{L}_{n} .
$$

As can be seen from (10) and (11), the dual function can be obtained by decomposing into $N$ independent subproblems as in (11) and solving them independently. It can be easily seen that the problem in (11) is concave. Thus, the optimal power allocation $P_{n}^{*}$ must satisfy $\frac{\partial \mathcal{L}_{n}}{\partial P_{n}^{*}}=0$, that is 


$$
\frac{\sum_{j=1}^{\varphi(M)} \frac{G_{M, j}}{2} \sqrt{\frac{g_{M, j} g_{s s}^{n}}{2 \pi\left(\sigma^{2}+g_{p s}^{n} T_{n}\right) P_{n}^{*}}} e^{-\frac{g_{M, j} g_{s s}^{n} P_{n}^{*}}{2\left(\sigma^{2}+g_{p s}^{n} T_{n}\right)}}}{1-\sum_{j=1}^{\varphi(M)} G_{M, j} Q\left(\sqrt{\frac{g_{M, j} g_{s s}^{n} P_{n}^{*}}{\sigma^{2}+g_{p s}^{n} T_{n}}}\right)}-\lambda g_{s p}^{n}-\mu=0 .
$$

We reformulate (12) as

$$
\begin{aligned}
\sum_{j=1}^{\varphi(M)} \frac{G_{M, j}}{2} \sqrt{\frac{g_{M, j} g_{s s}^{n}}{2 \pi\left(\sigma^{2}+g_{p s}^{n} T_{n}\right)}} e^{-\frac{g_{M, j} g_{s s}^{n} P_{n}^{*}}{2\left(\sigma^{2}+g_{p s}^{n} T_{n}\right)}}= & \left(1-\sum_{j=1}^{\varphi(M)} G_{M, j} Q\left(\sqrt{\frac{g_{M, j} g_{s s}^{n} P_{n}^{*}}{\sigma^{2}+g_{p s}^{n} T_{n}}}\right)\right) \\
& \times\left(\lambda g_{s p}^{n}+\mu\right) \sqrt{P_{n}^{*}} .
\end{aligned}
$$

It is noted that the closed-form expression for $P_{n}^{*}$ is not available from (13). However, it is easy to observe that the left-hand side of (13) is a monotonically decreasing function of $P_{n}$ and the right-hand side of (13) is a monotonically increasing function of $P_{n}$. Thus, we resort to the bisection search method to obtain $P_{n}^{*}$ for fixed $\lambda$ and $\mu$ as shown in Algorithm 1 (Alg1).

Algorithm 1: The bisection search method to obtain the optimal power allocation on subcarrier $n$.

1: Initialize $P_{n}^{\min }=0, P_{n}^{\max }=\bar{P}$.

2: If $\sum_{j=1}^{\varphi(M)} \frac{G_{M, j}}{2} \sqrt{\frac{g_{M, j} g_{s s}^{n}}{2 \pi\left(\sigma^{2}+g_{p s}^{n} T_{n}\right)}} e^{-\frac{g_{M, j} g_{s s}^{n} P_{n}^{\min }}{2\left(\sigma^{2}+g_{p s}^{n} T_{n}\right)}}<\left(1-\sum_{j=1}^{\varphi(M)} G_{M, j} Q\left(\sqrt{\frac{g_{M, j} g_{s s}^{n} P_{n}^{\min }}{\sigma^{2}+g_{p s}^{n} T_{n}}}\right)\right)\left(\lambda g_{s p}^{n}+\right.$ 4) $\sqrt{P_{n}^{\min }}$, then

3: $\quad P_{n}^{*}=P_{n}^{\min }$.

4: else if

$$
\begin{aligned}
& \sum_{j=1}^{\varphi(M)} \frac{G_{M, j}}{2} \sqrt{\frac{g_{M, j} g_{s s}^{n}}{2 \pi\left(\sigma^{2}+g_{p s}^{n} T_{n}\right)}} e^{-\frac{g_{M, j} g_{s s}^{n} P_{n}^{\max }}{2\left(\sigma^{2}+g_{p s}^{n} T_{n}\right)}}>\left(1-\sum_{j=1}^{\varphi(M)} G_{M, j} Q\left(\sqrt{\frac{g_{M, j} g_{s s}^{n} P_{n}^{\max }}{\sigma^{2}+g_{p s}^{n} T_{n}}}\right)\right) \\
& \left(\lambda g_{s p}^{n}+\mu\right) \sqrt{P_{n}^{\max }}, \text { then }
\end{aligned}
$$

5: $\quad P_{n}^{*}=P_{n}^{\max }$.

6: else

7: $\quad$ Repeat

8: $\quad P_{n}^{*}=\frac{P_{n}^{\min }+P_{n}^{\max }}{2}$.

9: $\quad$ If $\sum_{j=1}^{\varphi(M)} \frac{G_{M, j}}{2} \sqrt{\frac{g_{M, j} g_{s s}^{n}}{2 \pi\left(\sigma^{2}+g_{p s}^{n} T_{n}\right)}} e^{-\frac{g_{M, j} g_{s P_{n}^{n}}^{*}}{2\left(\sigma^{2}+g_{p s}^{n} T_{n}\right)}}>(1$

$$
\left.-\sum_{j=1}^{\varphi(M)} G_{M, j} Q\left(\sqrt{\frac{g_{M, j} g_{s s}^{n} P_{n}^{*}}{\sigma^{2}+g_{p s}^{n} T_{n}}}\right)\right)\left(\lambda g_{s p}^{n}+\mu\right) \sqrt{P_{n}^{*}} \text {, then }
$$

10: $\quad P_{n}^{\min }=P_{n}^{*}$.

11: $\quad$ else

12: : $\quad P_{n}^{\max }=P_{n}^{*}$.

13: $\quad$ End if

14: $\quad$ Until $\left|P_{n}^{\max }-P_{n}^{\min }\right| \leq \varepsilon$.

\section{5: End if}

where $\epsilon>0$ denotes the error tolerance. 
Finally, the optimal $\lambda$ and $\mu$ can be obtained by the subgradient method that iteratively updates $\lambda$ and $\mu$ as follows

$$
\begin{gathered}
\lambda(t+1)=\lambda(t)-\theta_{1}\left(\bar{I}-\sum_{n=1}^{N} P_{n}^{*} g_{s p}^{n}\right), \\
\mu(t+1)=\mu(t)-\theta_{2}\left(\bar{P}-\sum_{n=1}^{N} P_{n}^{*}\right),
\end{gathered}
$$

where $t$ is the iteration number, $\theta_{1}$ and $\theta_{2}$ are the step sizes. The subgradient update in (14) and (15) is guaranteed to converge to the optimal $\lambda$ and $\mu$ as long as $\theta_{1}$ and $\theta_{2}$ are chosen to be sufficiently small [30].

The algorithm to solve P1 is summarized as follows.

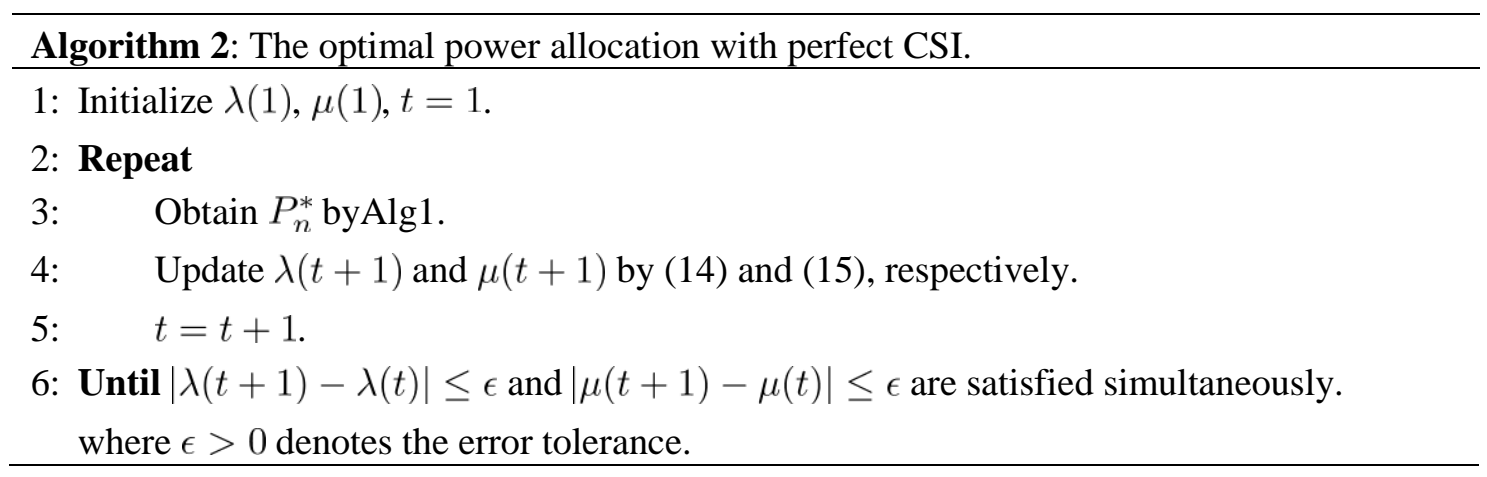

The complexity of Algorithm 2 (Alg2) is analyzed briefly as follows. For fixed $\lambda$ and $\mu$, the bisection method in Alg1 converges as $\mathcal{O}\left(N \log _{2}(1 / \varepsilon)\right)$ with guaranteed error tolerance of $\varepsilon$. As for the subgradient method, a small number $\Omega$ of iterations are required according to [31]. In summary, the total complexity is $\mathcal{O}\left(\Omega N \log _{2}(1 / \varepsilon)\right)$.

In order to reduce the complexity of $\mathrm{Alg} 2$, in the rest of the section, we propose a suboptimal algorithm with less complexity. According to Table 6.1 in [32], the expression in (4) can be approximated at high SINR as

$$
\operatorname{ber}\left(\gamma_{n}\right) \approx a Q\left(\sqrt{b \gamma_{n}}\right),
$$

where $\quad a=4(\sqrt{M}-1) /\left(\sqrt{M} \log _{2} M\right) \quad$ and $\quad b=3 \log _{2} M /(M-1) . \quad$ The above approximation has been widely used in literature such as [21]. Thus, P2 can be approximated as

$$
\begin{aligned}
& \mathrm{P} 3: \max _{P_{n} \geq 0} \sum_{n=1}^{N} \ln \left(1-a Q\left(\sqrt{b \gamma_{n}}\right)\right) \\
& \text { s.t. constraints }(2),(3) .
\end{aligned}
$$

Under the assumption of high SINR, the value of $a Q\left(\sqrt{b \gamma_{n}}\right)$ is small and the objective function in (17) can be approximated as $-\sum_{n=1}^{N} a Q\left(\sqrt{b \gamma_{n}}\right)$. It has been verified in [21] that $-a Q\left(\sqrt{b \gamma_{n}}\right)$ is concave. Thus, $-\sum_{n=1}^{N} a Q\left(\sqrt{b \gamma_{n}}\right)$ is also concave. Using the method similar to that in solving $\mathrm{P} 2$, the optimal power allocation, $P_{n}^{*}$, for $\mathrm{P} 3$, satisfies 


$$
-\frac{a}{2} \sqrt{\frac{b g_{s s}^{n}}{2 \pi\left(\sigma^{2}+g_{p s}^{n} T_{n}\right) P_{n}^{*}}} e^{-\frac{b g_{s S}^{n} P_{n}^{*}}{2\left(\sigma^{2}+g_{p s}^{n} T_{n}\right)}}+\lambda g_{s p}^{n}+\mu=0 .
$$

After some mathematical manipulation of (18), we have

$$
\frac{b g_{s s}^{n} P_{n}^{*}}{\sigma^{2}+g_{p s}^{n} T_{n}} e^{\frac{b g_{s s}^{n} P_{n}^{*}}{\sigma^{2}+g_{p s}^{n} T_{n}}}=\frac{a^{2} b^{2}\left(g_{s s}^{n}\right)^{2}}{8 \pi\left(\sigma^{2}+g_{p s}^{n} T_{n}\right)^{2}\left(\lambda g_{s p}^{n}+\mu\right)^{2}} .
$$

It is observed that (19) has a form $x e^{x}=a$. Thus, the optimal power allocation, $P_{n}^{*}$, for P3, can be obtained in closed-form as

$$
P_{n}^{*}=\frac{\sigma^{2}+g_{p s}^{n} T_{n}}{b g_{s s}^{n}} \mathcal{W}\left(\frac{a^{2} b^{2}\left(g_{s s}^{n}\right)^{2}}{8 \pi\left(\sigma^{2}+g_{p s}^{n} T_{n}\right)^{2}\left(\lambda g_{s p}^{n}+\mu\right)^{2}}\right),
$$

where $\mathcal{W}(\cdot)$ denotes the Lambert $\mathrm{W}$ function [33]. The value of $\lambda$ and $\mu$ can be obtained by the subgradient method similar to that in Alg2.

The suboptimal algorithm is summarized as follows.

Algorithm 3: The suboptimal power allocation with perfect CSI.

1: Initialize $\lambda(1), \mu(1), t=1$.

2: Repeat

3: $\quad$ Calculate $P_{n}^{*}$ by (20).

4: $\quad$ Update $\lambda(t+1)$ and $\mu(t+1)$ by (14) and (15), respectively.

5: $\quad t=t+1$.

6: Until $|\lambda(t+1)-\lambda(t)| \leq \epsilon$ and $|\mu(t+1)-\mu(t)| \leq \epsilon$ are satisfied simultaneously.

Since Algorithm 3 (Alg3) does not need to perform the bisection search, the complexity reduces to $\mathcal{O}(\Omega N)$, which is much less complex than $\mathcal{O}\left(\Omega N \log _{2}(1 / \varepsilon)\right)$ of $A \lg 2$.

\section{Power Allocation with CDI}

In this section, with only the CDI of the interference links available at the SU, the power allocation problem for the SU to minimize the aggregate BER is studied. We assume that the interference links experience Rayleigh fading and thus the channel power gains $g_{s p}^{n}$ and $g_{p s}^{n}$ follow exponential distribution. We denote $\alpha_{s p}$ and $\alpha_{p s}$ the mean values of $g_{s p}^{n}$ and $g_{p s}^{n}$, respectively. It is noted that it is impossible for the SU to satisfy the interference power constraint (2) with only CDI of $g_{s p}^{n}$. Thus, instead of using the interference power constraint, the interference outage constraint, which allows a certain percentage of outage, is adopted as

$$
P_{\text {out }}=\operatorname{Pr}\left\{\sum_{n=1}^{N} g_{s p}^{n} P_{n} \geq \bar{I}\right\} \leq \zeta,
$$

where $\zeta$ is the target interference outage probability. Note that, although a certain percentage of interference outage exists, the PU can be protected as long as the constraint (21) is satisfied, and such kind of constraint has been adopted in existing literature such as [34], [35].

The power allocation problem to minimize the aggregate BER under the interference outage constraint and the transmit power constraint with CDI can be formulated as follows 


$$
\mathrm{P} 4: \min _{P_{n} \geq 0} 1-\prod_{n=1}^{N}\left(1-\operatorname{ber}\left(\gamma_{n}^{\prime}\right)\right)
$$

s.t. constraints $(3),(21)$.

where $\gamma_{n}^{\prime}=g_{s s}^{n} P_{n} /\left(\sigma^{2}+\alpha_{p s} T_{n}\right)$. Here, we use the mean value of $g_{p s}^{n}$ instead of $g_{p s}^{n}$ due to the fact that $g_{p s}^{n}$ is not available. To solve P4, we need to obtain the distribution of $\sum_{n=1}^{N} g_{s p}^{n} P_{n}$ to further investigate constraint (21). For this, we use the result from [36] where the probability density function of $I=\sum_{n=1}^{N} g_{s p}^{n} P_{n}$ is given as

$$
f_{I}(x)=\left(\prod_{n=1}^{N} \frac{1}{\alpha_{s p} P_{n}}\right) \sum_{n=1}^{N} \frac{e^{-\frac{x}{\alpha_{s p} P_{n}}}}{\prod_{j=1, j \neq n}^{N}\left(\frac{1}{\alpha_{s p} P_{j}}-\frac{1}{\alpha_{s p} P_{n}}\right)} .
$$

Thus, the interference outage probability can be written as

$$
\begin{aligned}
P_{\text {out }} & =\int_{\bar{I}}^{\infty} f_{I}(x) d x \\
& =\left(\prod_{n=1}^{N} \frac{1}{\alpha_{s p} P_{n}}\right) \sum_{n=1}^{N} \frac{\alpha_{s p} P_{n} e^{-\frac{\bar{I}}{\alpha_{s p} P_{n}}}}{\prod_{j=1, j \neq n}^{N}\left(\frac{1}{\alpha_{s p} P_{j}}-\frac{1}{\alpha_{s p} P_{n}}\right)} .
\end{aligned}
$$

Even though we have obtained the closed-form expression for $P_{\text {out }}$, it is still difficult to solve P4. Alternatively, we develop a heuristic power allocation algorithm in what follows. First, we consider a similar problem without constraint (21) as

$$
\begin{gathered}
\mathrm{P} 5: \min _{P_{n} \geq 0} 1-\prod_{n=1}^{N}\left(1-\operatorname{ber}\left(\gamma_{n}^{\prime}\right)\right) \\
\text { s.t. constraints }(3) .
\end{gathered}
$$

Following the similar methods for solving P1 in section 3, we can obtain optimal algorithm for solving P5 similar to Alg2 and suboptimal algorithm similar to Alg3. We denote Alg2 and Alg3 for solving P5 as Alg2 ${ }^{\prime}$ and $\mathrm{Alg} 3^{\prime}$, respectively $^{2}$.

Proposition 1: $P_{\text {out }}$ is a strictly decreasing function of the dual variable $\mu$ if $\mathrm{Alg} 2^{\prime}$ or $\mathrm{Alg} 3^{\prime}$ is applied.

Proof: If $\mu$ increases, the right-hand side of (13) increases which results in smaller value of $P_{n}$. By examining (21), it is easy to see that $P_{\text {out }}$ is an increasing function of $P_{n}$. Therefore, $P_{\text {out }}$ is a strictly decreasing function of $\mu$ if $\mathrm{Alg} 2^{\prime}$ is applied. As for $\mathrm{Alg} 3^{\prime}$, since $\mathcal{W}(x)$ is a increasing function of $x$ for $x \geq 0$ [33], $P_{n}$ is a decreasing function of $\mu$ according to (20). Thus, $P_{\text {out }}$ is a strictly decreasing function of $\mu$ if $\mathrm{Alg} 3{ }^{\prime}$ is applied.

This completes the proof.

Based on Proposition 1, a simple bisection search method can be used to determine the $\mu$ until the target interference outage probability is achieved. The proposed power allocation algorithm to solve P4 is described in Algorithm 4.

\footnotetext{
${ }^{2}$ Compared to P1, the constraint (2) is not considered in P5. Thus, the dual variable $\lambda$ in Alg2 and Alg3 is omitted in $\mathrm{Alg} 2^{\prime}$ and $\operatorname{Alg} 3^{\prime}$. Besides, to obtain $P_{n}^{*}$ in $\operatorname{Alg} 2^{\prime}$, the value of $\lambda$ in $\operatorname{Alg} 1$ is set to zero.
} 


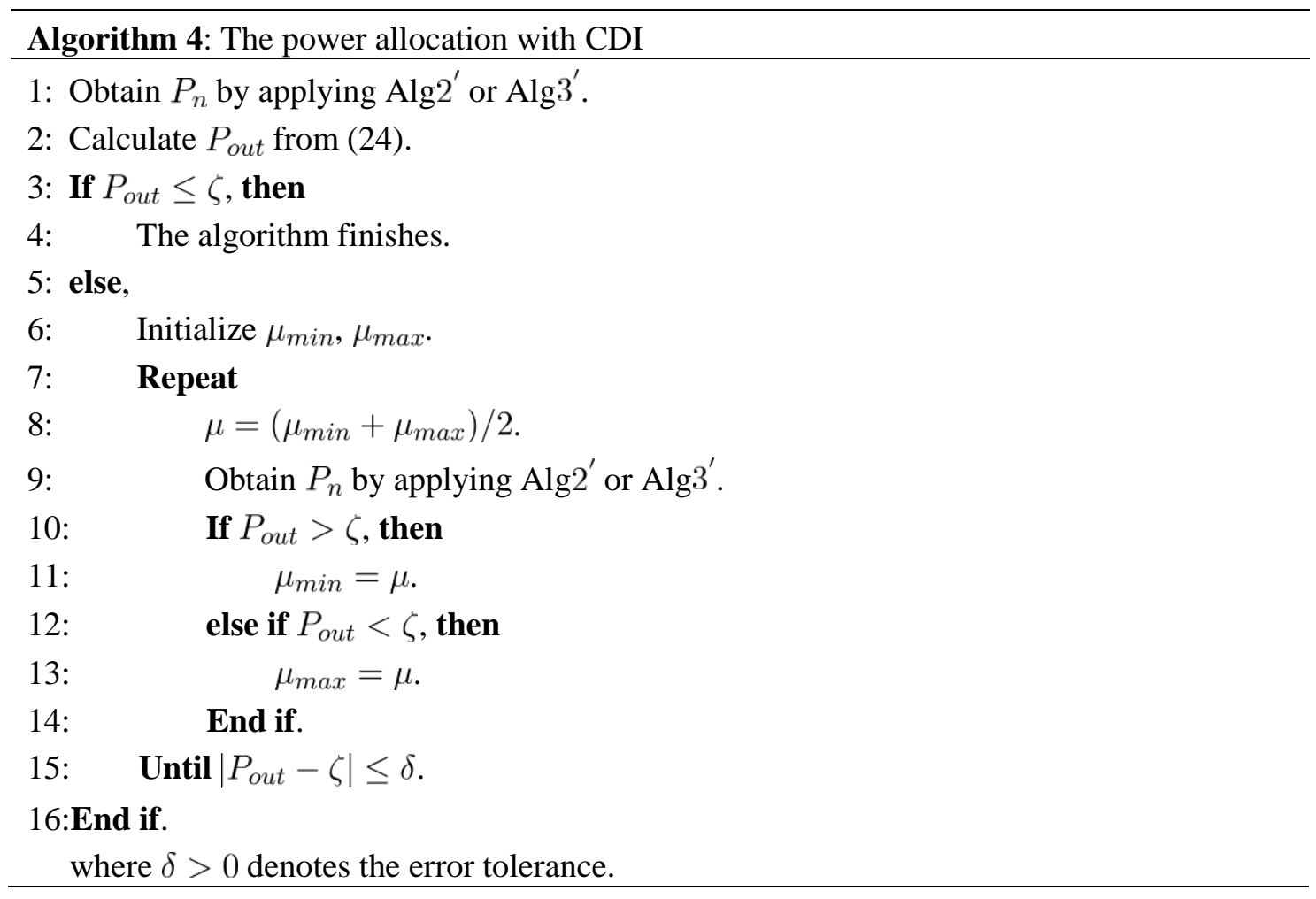

We denote Algorithm 4 which uses Alg2 ${ }^{\prime}$ to obtain $P_{n}^{*}$ as Alg4-1 and denote Algorithm 4 which uses Alg3 ${ }^{\prime}$ to obtain $P_{n}^{*}$ as Alg4-2. The complexity of Alg4-1 and Alg4-2 is analyzed briefly as follows. Under the worst case when bisection search method for obtaining $\mu$ is involved, the bisection method converges as $\mathcal{O}\left(\log _{2}(1 / \delta)\right)$. According to the analysis in Section 3, the complexity of Alg2 is $\mathcal{O}\left(\Omega N \log _{2}(1 / \varepsilon)\right)$ and the complexity of $A \lg 3$ is $\mathcal{O}(\Omega N)$. Therefore, the total complexity of Alg4-1 is $\mathcal{O}\left(\left(1+\log _{2}(1 / \delta)\right) \Omega N \log _{2}(1 / \varepsilon)\right)$ and the total complexity of $\operatorname{Alg} 4-2 \mathcal{O}\left(\left(1+\log _{2}(1 / \delta)\right) \Omega N\right)$.

\section{Simulation Results}

This section presents simulation examples to verify the performance of the proposed power allocation algorithms. In the simulation, all the channels involved are assumed to be Rayleigh fading. The average channel power gain for the secondary communication link is assumed to be 1, i.e. $E\left\{g_{s s}^{n}\right\}=1, \forall n$. The average channel power gains for the interference links are assumed to be 0.001 , i.e. $E\left\{g_{s p}^{n}\right\}=0.001, E\left\{g_{p s}^{n}\right\}=0.001, \forall n$. In addition, we set $\sigma^{2}=0.001, M=4, N=8, T_{n}=0 \mathrm{~dB}$ and $\bar{P}=10 \mathrm{~dB}$.

Fig. 1 plots the aggregate BER of the SU with Perfect CSI against $\bar{I}$. In the figure, the results of three reference algorithms are also given for comparison purposes. In reference algorithm 1 (Ref1), the uniform power allocation, where the available transmit power $\bar{P}$ and interference power $\bar{I}$ are distributed evenly between $N$ subcarriers, is used, i.e., $P_{n}=\min \left(\bar{P}, \bar{I} / g_{s p}^{n}\right) / N$. The reference algorithm 2 (Ref2) adopts the conventional water-filling power allocation for non-CR OFDM systems [26]. Beside, to control the 
interference to the PU, the power allocation on subcarrier $n$ is capped by $\bar{I} / N g_{s p}^{n}$. In reference algorithm 3 (Ref3), the optimal power allocation algorithm to maximize the SU sum rates proposed in [5] is used. It is seen that the aggregate BER of the SU decreases with the increases of $\bar{I}$ and finally saturates as $\bar{I}$ increases further. This indicates that $\bar{P}$ restricts the performance of the SU when $\bar{I}$ is large. It is also seen that the aggregate BER achieved by Alg2 is smaller than that achieved by Alg3. In addition, it is observed that the three reference algorithms achieve very similar aggregate BER levels for small $\bar{I}$. For large $\bar{I}$, it is observed that Ref1 achieves lower aggregate BER than that of Ref2 and Ref3. It is also observed that both $A \lg 2$ and $A \lg 3$ significantly outperform the three reference algorithms, especially for large $\bar{I}$.

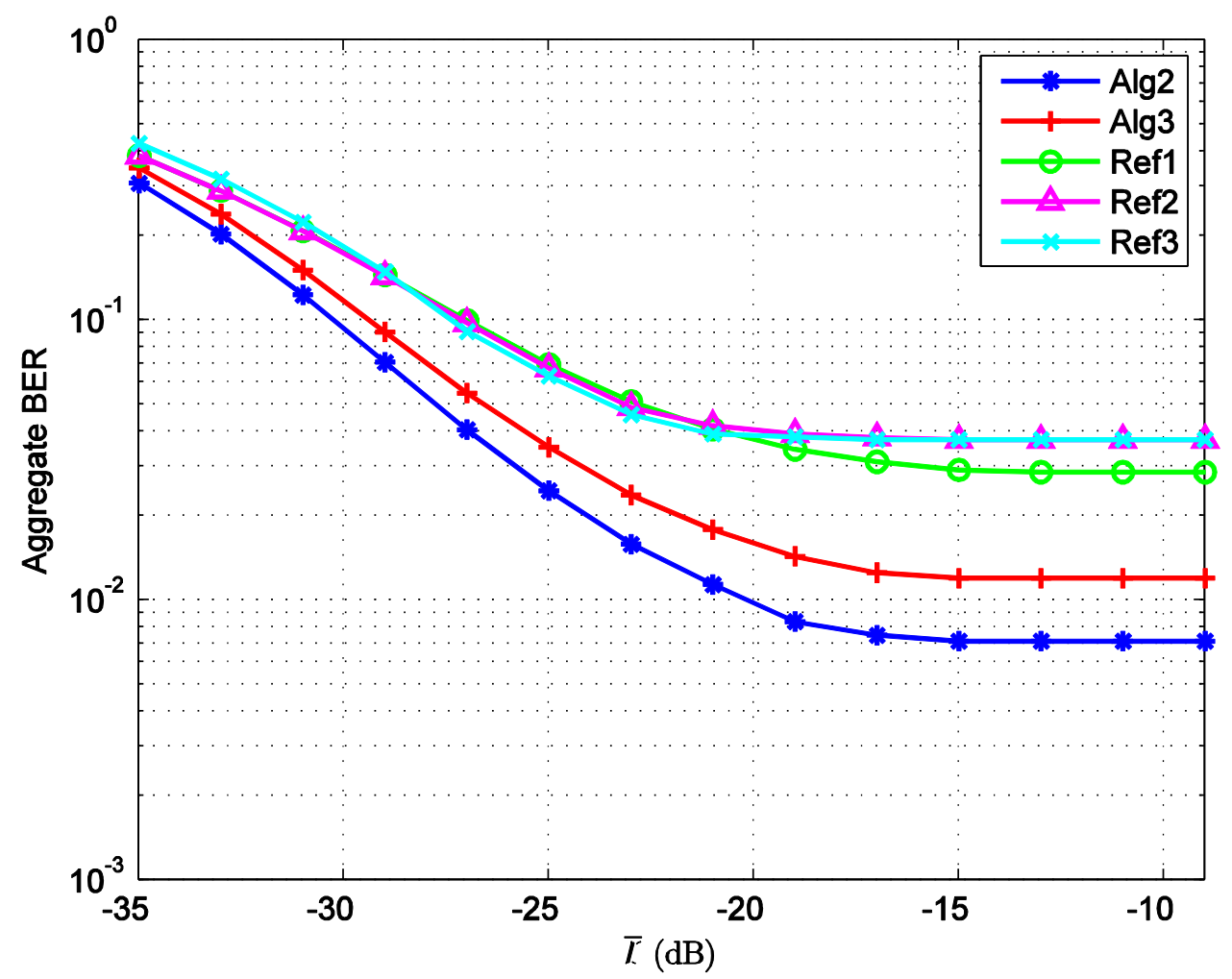

Fig. 1. Aggregate BER of the SU with Perfect CSI against $\bar{I}$.

Fig. 2 plots the convergence behavior of the proposed power allocation algorithms with CDI. It is shown that both Alg4-1 and Alg4-2 converge in less than twenty iterations. This indicates that the proposed heuristic algorithms are practically feasible. It is also shown that the interference outage probability $P_{\text {out }}$ equals to the target interference outage probability $\zeta$ when the algorithms converge. This indicates that the proposed algorithms can guarantee that the interference outage constraint in (21) is satisfied and thus the PU is protected with only CDI available. 


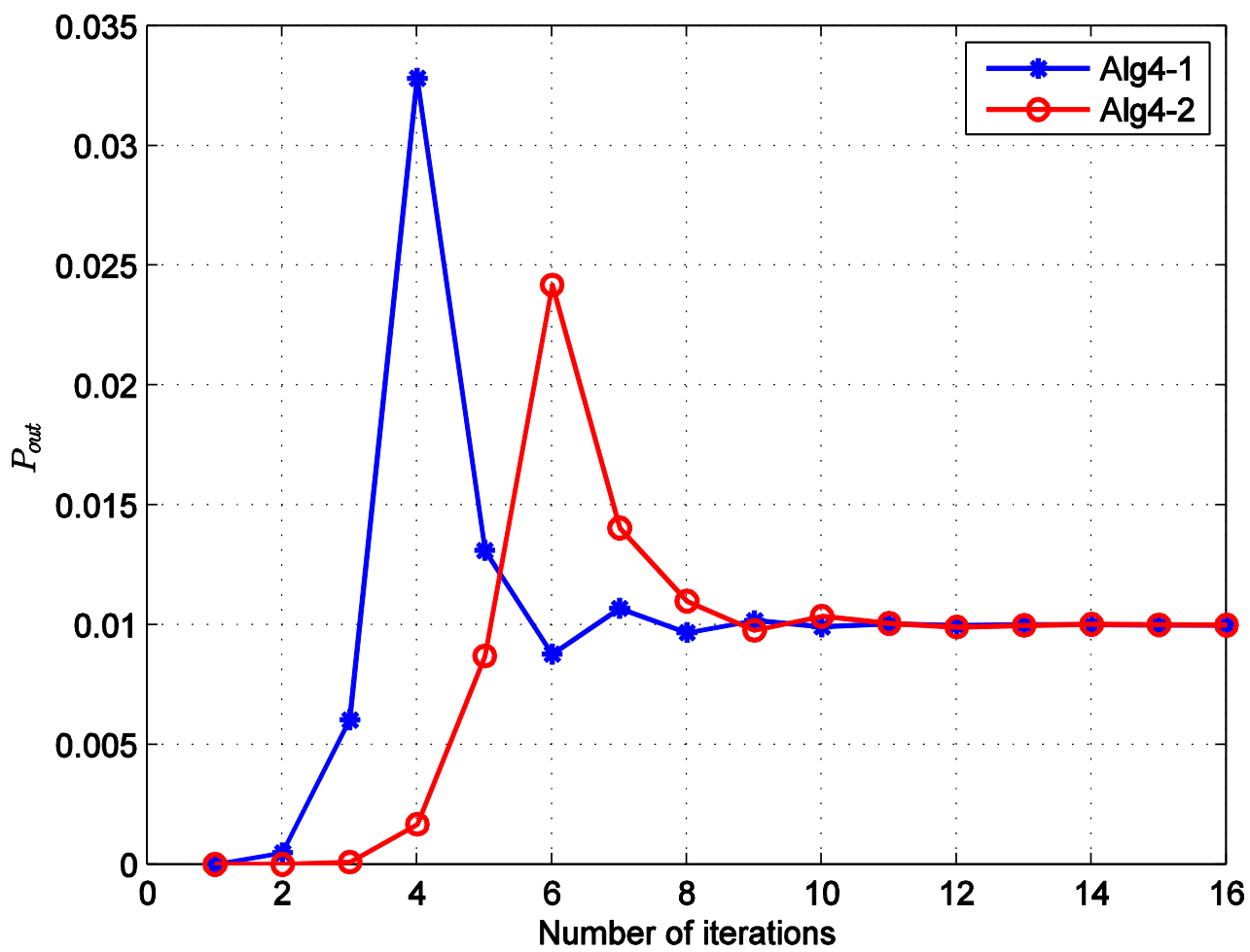

Fig. 2. Convergence behavior of the proposed power allocation algorithms with $\mathrm{CDI}(\bar{I}=-20 \mathrm{~dB}$ and $\zeta=0.01)$.

Fig. 3 plots the aggregate BER of the SU with CDI against $\bar{I}$ for different values of $\zeta$. For the purpose of comparison, results of $\mathrm{Alg} 2$ and $\mathrm{Alg} 3$ with perfect CSI are also given. It is seen that the aggregate BER of the SU with CDI achieved by Alg4-1 (or Alg4-2) decreases as $\bar{I}$ increases and saturates when $\bar{I}$ is very large. It is also seen that, as $\zeta$ increases, the aggregate BER decreases for small $\bar{I}$ and does not change for large $\bar{I}$. This indicates that, for large value of $\bar{I}$, the transmit power constraint $\bar{P}$ becomes the dominant constraint and thus increasing the values of $\bar{I}$ and $\zeta$ does not improve the performance of the SU. In addition, it is seen that Alg4-1 outperforms Alg4-2. It is also seen that Alg4-1 and Alg4-2 always perform worst than Alg2 and Alg3, respectively. This indicates that the performance of the SU drops with only CDI available compared to the case of perfect CSI. 


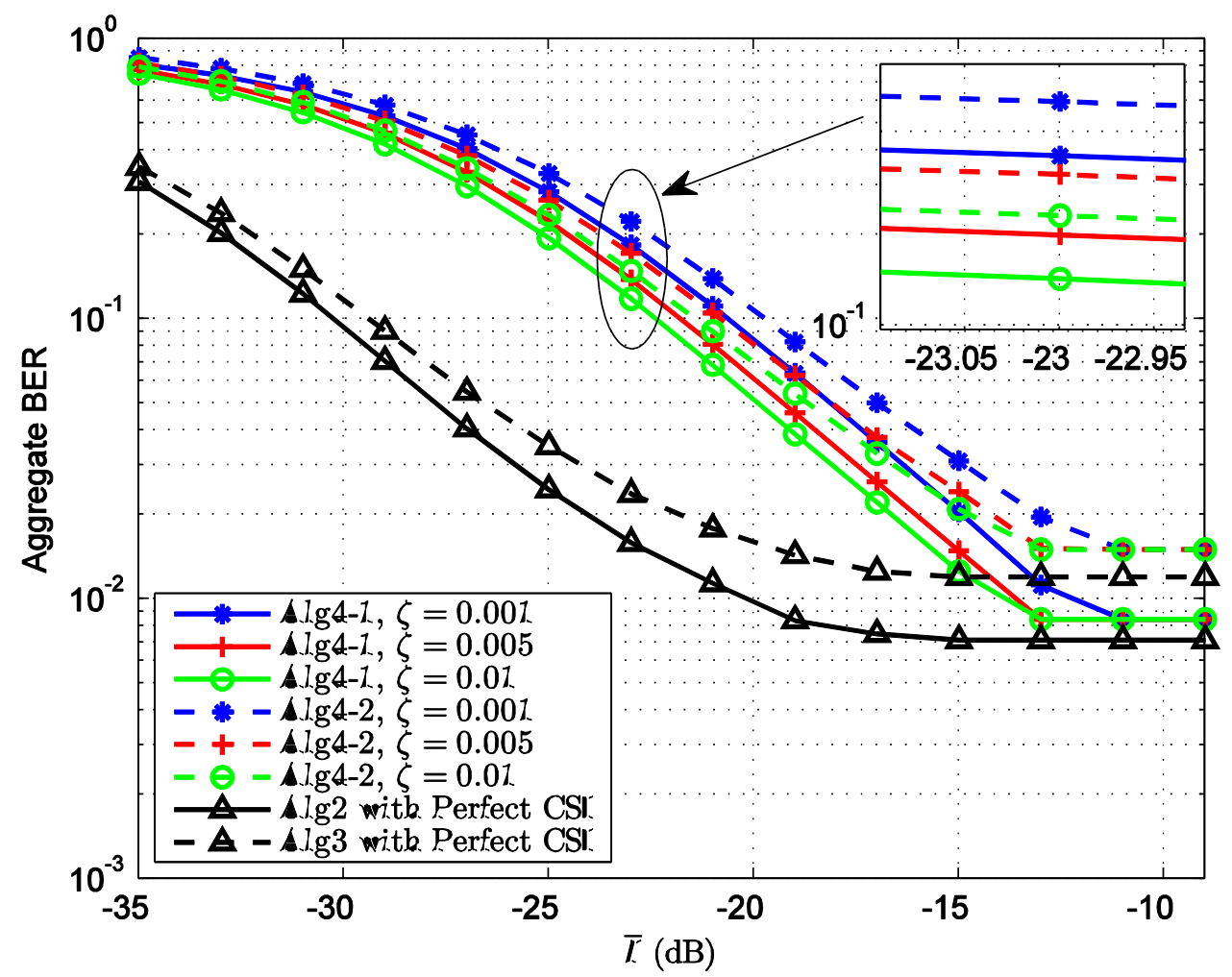

Fig. 3. Aggregate BER of the SU with CDI against $\bar{I}$.

\section{Conclusions}

This paper studies the power allocation problem in a downlink OFDM-based CR network targeting at minimizing the aggregate BER of the SU. Assuming that the instantaneous CSI of the interference links is perfectly known, an optimal algorithm and a suboptimal algorithm with less complexity are proposed under the interference power constraint and the transmit power constraint. It is shown that the proposed algorithms significantly outperform the existing reference algorithms. On the other hand, assuming only the CDI of the interference links is available, two heuristic power allocation algorithms based on bisection search method are proposed under the interference outage constraint and the transmit power constraint. It is shown that the PU QoS in terms of interference outage probability can be well protected by the proposed algorithms with only CDI available. It is also shown that with only CDI available, the performance of the SU drops compared to the case of perfect CSI.

\section{References}

[1] Y.-C. Liang, K.-C. Chen, G. Y. Li, and P. Mahonen, "Cognitive radio networking and communications: An overview," IEEE Transactions on Vehicular Technology, vol. 60, no. 7, pp. 3386-3407, 2011. Article (CrossRef Link)

[2] A. Goldsmith, S. A. Jafar, I. Maric, and S. Srinivasa, "Breaking spectrum gridlock with cognitive radios: An information theoretic perspective," Proceedings of the IEEE, vol. 97, no. 5, pp. 894-914, 2009. Article (CrossRef Link) 
[3] B. Zhong, Z. Zhang, X. Zhang, J. Wang, and K. Long, "Partial relay selection with fixed-gain relays and outdated CSI in underlay cognitive networks," IEEE Transactions on Vehicular Technology, vol. 62, no. 9, pp. 4696-4701, 2013. Article (CrossRef Link)

[4] G. Bansal, M. J. Hossain, and V. K. Bhargava, "Optimal and suboptimal power allocation schemes for OFDM-based cognitive radio systems," IEEE Transactions on Wireless Communications, vol. 7, no. 11, pp. 4710-4718, 2008. Article (CrossRef Link)

[5] Y. Zhang and C. Leung, "An efficient power-loading scheme for OFDM-based cognitive radio systems," IEEE Transactions on Vehicular Technology, vol. 59, no. 4, pp. 1858-1864, 2010. Article (CrossRef Link)

[6] M. Chowdhury, A. Singla, and A. K. Chaturvedi, "A family of power allocation schemes achieving high secondary user rates in spectrum sharing OFDM cognitive radio," in Proc. of IEEE Global Communications Conference (GLOBECOM), pp. 1144-1149, 2012. Article (CrossRef Link)

[7] W.-C. Pao and Y.-F. Chen, "Constant interference methods for power allocation in orthogonal frequency division multiplexing-based cognitive radio networks," IET Communications, vol. 6, no. 18, pp. 3204-3212, 2012. Article (CrossRef Link)

[8] X. Ling, B. Wu, H. Wen, L. Pan, and F. Luo, "Fast and efficient parallel-shift water-filling algorithm for power allocation in orthogonal frequency division multiplexing-based underlay cognitive radios," IET Communications, vol. 7, no. 12, pp. 1269-1278, 2013.

Article (CrossRef Link)

[9] W. Pao and Y. Chen, "Adaptive gradient-based methods for adaptive power allocation in OFDM-based cognitive radio networks," IEEE Transactions on Vehicular Technology, vol. 63, no. 2, pp. 836-848, 2014. Article (CrossRef Link)

[10] X. Kang, H. K. Garg, Y.-C. Liang, and R. Zhang, "Optimal power allocation for OFDM-based cognitive radio with new primary transmission protection criteria," IEEE Transactions on Wireless Communications, vol. 9, no. 6, pp. 2066-2075, 2010. Article (CrossRef Link)

[11] D. Xu, Z. Feng, and P. Zhang, "Power allocation schemes for downlink cognitive radio networks with opportunistic sub-channel access." KSII Transactions on Internet \& Information Systems, vol. 6, no. 7, pp. 1777-1791, 2012. Article (CrossRef Link)

[12] Z. Zhu, J. Peng, P. Yao, F. Jiang, and K. Lin, "A worst-case robust distributed power allocation scheme for OFDM-based cognitive radio networks," in Proc. of IEEE International Conference on Communications (ICC), pp. 1489-1494, 2014. Article (CrossRef Link)

[13] D. Xu and Q. Li, "Power allocation for two-way OFDM-based spectrum sharing cognitive radio networks," IEICE Transactions on Fundamentals of Electronics, Communications and Computer Sciences, vol. 98, no. 3, pp. 918-922, 2015. Article (CrossRef Link)

[14] R. Khederzadeh and H. Farrokhi, "Optimal and suboptimal adaptive algorithms for rate and power transmission in OFDM-based cognitive radio systems," Computers \& Electrical Engineering, vol. 42, pp. 168-177, 2015. Article (CrossRef Link)

[15] D. Jiang, Z. Xu, W. Li, and Z. Chen, "Topology control-based collaborative multicast routing algorithm with minimum energy consumption," International Journal of Communication Systems, 2014, [Online]. Available: http://dx.doi.org/10.1002/dac.2905. Article (CrossRef Link)

[16] D. Jiang, Z. Xu, W. Li, and Z. Chen, "Network coding-based energy-efficient multicast routing algorithm for multi-hop wireless networks," Journal of Systems and Software, vol. 104, pp. 152-165, 2015. Article (CrossRef Link)

[17] D. Jiang, Z. Xu, J. Liu, and W. Zhao, "An optimization-based robust routing algorithm to energy-efficient networks for cloud computing," Telecommunication Systems, pp. 1-10, 2015. Article (CrossRef Link)

[18] Y. Huang, J. Wang, Q. Wu, C. Zhong, and C. Li, "Outage performance of spectrum sharing systems with MRC diversity under multiple primary user's interference," IEEE communications letters, vol. 18, no. 4, pp. 576-579, 2014. Article (CrossRef Link)

[19] D. Xu and Q. Li, "Joint power and rate allocation in cognitive radio multicast networks for outage probability minimization," IEICE Transactions on Fundamentals of Electronics, Communications and Computer Sciences, vol. 97, no. 3, pp. 904-906, 2014. Article (CrossRef Link) 
[20] D. Xu, Z. Feng, and P. Zhang, "Minimum average BER power allocation for fading channels in cognitive radio networks," in Proc. of IEEE Wireless Communications and Networking Conference (WCNC), pp. 78-83, 2011. Article (CrossRef Link)

[21] M. R. Mili and K. A. Hamdi, "Minimum BER analysis in interference channels," IEEE Transactions on Wireless Communications, vol. 12, no. 7, pp. 3191-3201, 2013. Article (CrossRef Link)

[22] D. Li, "Performance analysis of uplink cognitive cellular networks with opportunistic scheduling," IEEE Communications Letters, vol. 14, no. 9, pp. 827-829, 2010. Article (CrossRef Link)

[23] T. Xu, J. Ge, and H. Ding, “Opportunistic scheduling for uplink cognitive cellular networks with outage protection of the primary user," IEEE communications letters, vol. 17, no. 1, pp. 71-74, 2013. Article (CrossRef Link)

[24] Y. Wang and J. Coon, "BER minimization for cognitive radio systems with difference antenna selection," in Proc. of IEEE Pacific Rim Conference on Communications, Computers and Signal Processing (PacRim), pp. 304-309, 2011. Article (CrossRef Link)

[25] K. Ho-Van and P. Sofotasios, "Exact bit-error-rate analysis of underlay decode-andforward multi-hop cognitive networks with estimation errors," IET Communications, vol. 7, no. 18, pp. 2122-2132, December, 2013. Article (CrossRef Link)

[26] T. M. Cover and J. A. Thomas, Elements of information theory. John Wiley \& Sons, 2012.

[27] D. Xu, Y. Li, Z. Feng, and P. Zhang, "Resource allocation for multiuser cognitive radio with primary user's cooperation," in Proc. of IEEE Global Communications Conference (GLOBECOM) Workshops, pp. 1419-1423, 2011. Article (CrossRef Link)

[28] B. Choi and L. Hanzo, "Optimum mode-switching-assisted constant-power single-and multicarrier adaptive modulation," IEEE Transactions on Vehicular Technology, vol. 52, no. 3, pp. 536-560, 2003. Article (CrossRef Link)

[29] S. Boyd and L. Vandenberghe, "Convex Optimization. Cambridge," U.K.: Cambridge Univ. Press, 2004. Article (CrossRef Link)

[30] D. Bertsekas, W. Hager, and O. Mangasarian, "Nonlinear programming. Athena Scientific Belmont," MA, 1999.

[31] D. Xu, Z. Feng, and P. Zhang, "Resource allocation for heterogeneous services in multiuser cognitive radio networks," International Journal of Communication Systems, vol. 27, no. 10, pp. 2121-2140, 2014. Article (CrossRef Link)

[32] A. Goldsmith, Wireless communications. Cambridge university press, 2005. Article (CrossRef Link)

[33] R. M. Corless, G. H. Gonnet, D. E. G. Hare, D. J. Jeffrey, and D. E. Knuth, "On the lambertw function," Advances in Computational Mathematics, vol. 5, no. 1, pp. 329-359, 1996. Article (CrossRef Link)

[34] D. Xu. and Q. Li, "On the outage capacity of fading cognitive multicast channel," IEICE Transactions on Fundamentals of Electronics, Communications and Computer Sciences, vol. 97 , no. 11, pp. 2272-2275, 2014. Article (CrossRef Link)

[35] D. Xu and Q. Li, "Ergodic capacity and outage probability optimization for secondary user in cognitive radio networks under interference outage constraint," AEU-International Journal of Electronics and Communications, vol. 68, no. 8, pp. 747-755, 2014. Article (CrossRef Link)

[36] M. Akkouchi, "On the convolution of exponential distributions," J. Chungcheong Math. Soc, vol. 21, no. 4, pp. 501-510, 2008. 


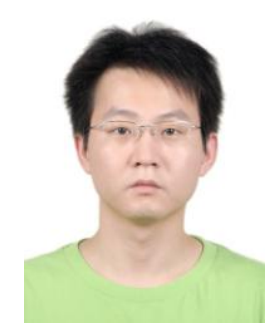

Ding $\mathrm{Xu}$ received the $\mathrm{PhD}$ degree in communication and information systems from Beijing University of Posts and Telecommunications, Beijing, China, in 2013. Before pursuing PhD, he worked as an engineer for Nortel Networks (China), and was responsible for developing automation testing tools for LTE equipment. Currently, he is a lecturer in Nanjing University of Posts and Telecommunications, Nanjing, China. His research interests include resource allocation and performance analysis for cognitive radio networks.

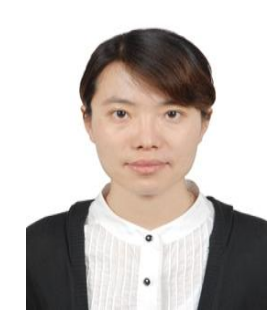

Qun Li received the $\mathrm{PhD}$ degree in signal and information processing from Beijing University of Posts and Telecommunications, Beijing, China, in 2013. She used to be a visiting student for one and a half year in the Center for Research in Intelligent Systems (VISLab), University of California, Riverside, CA, USA. Currently, she is a lecturer in Nanjing University of Posts and Telecommunications, Nanjing, China. Her research interests include signal processing in communication systems. 